Revista General de Información y Documentación ISSN: 1132-1873

http://dx.doi.org/10.5209/RGID.60805

\title{
Gaming como Instrumento Educativo para una Educación en Competencias Digitales desde los Academic Skills Centres
}

\author{
Miguel Ángel Marzal García-Quismondo'; Eduardo Cruz-Palacios ${ }^{2}$
}

Recibido: 22 de abril 2018 / Aceptado: 25 de noviembre de 2018

Resumen. Se analiza el modelo educativo competencial de la Educación Superior del siglo XXI desde la óptica de su pieza fundamental, las competencias adquiridas en el entorno de las multialfabetizaciones. La investigación argumenta el análisis en cuatro dimensiones: primero la Web como espacio educativo, elemento emergente en la generación de conocimiento, en el que plantear la innovación educativa como método y factor del comportamiento informacional, el Conectivismo como el modelo pedagógico idóneo para la educación ciberespacial, y las redes como medio apropiado de transmisión; luego, la competencia digital como objetivo didáctico prioritario para el aprendizaje permanente y cooperativo, atendiendo a sus marcos normativos de definición y aplicación; sigue el estudio del Gaming como objeto educativo y material didáctico web idóneo para programas educativos competenciales, debido a su efectividad pedagógica; por fin, la consideración de los Academic Skills Centres (ASC) como el espacio necesario para el correcto tratamiento del Gaming como objetos de aprendizaje y para el desarrollo de los programas didácticos competenciales. La investigación termina con una propuesta de cómo debería ser un programa educativo competencial en un ASC presentando su diseño instructivo, que es modular y progresivo, su dinámica docente como metodología didáctica para su mayor eficacia educativa, y el diseño de su programación didáctica estructurada en doce unidades didácticas.

Palabras clave: Educación Superior, Videojuegos, Competencias Digitales, Gaming, Academic Skills Centres; Multialfabetización; Objetos de Aprendizaje; Aprendizaje.

\section{[en] Gaming as an Educational Material for Digital Competences in Education from Academic Skills Centres}

\begin{abstract}
The competence-based educational model for 21st century Higher Education is analyzed taking into consideration how learners' competences thrive in the multiliteracies environment. There are four main arguments for this research. Firstly, the Web, as an educational space, is an essential system to allow the generation of knowledge, and that requires the methodology of educational innovation on informational behavior, Connectivism as the ideal pedagogy on non-borders learning (anytime and anywhere), and networks as the appropriate means of the communication of knowledge. Then, digital competences are considered as a priority to framework-based permanent, cooperative learning objectives. In addition, Gaming is examined as a suitable, web, educational resource that is potentially effective on learning programs. After that, Academic Skills Centres (ASC) are reckoned as

1 Universidad Carlos III de Madrid. Departamento de Biblioteconomía y Documentación

E-mail: mmarzal@bib.uc3m.es

2 Universidad Carlos III de Madrid. Departamento de Biblioteconomía y Documentación

E-mail:eduardocruzpalacios@gmail.com
\end{abstract}


a necessary institution from which Gaming-based learning objects are managed in order that they are available for the development of educational competences programs. Finally, this research ends up proposing a competence-based program that reflects how similar, educational programs taking place in ASCs must be designed regarding their modular, progressive structure consisting of twelve didactic units, and their teaching dynamics as pedagogical methodology geared towards a greater educational effectiveness.

Keywords: Higher Education, Videogames, Digital Competences, Gaming, Academic Skills Centres; Multiliteracies; Learning Objects; Learning.

Sumario. 1. Introducción. 2. La web como espacio educativo. 3. Competencia digital como instrumento educativo. 4. Gaming como objeto educativo. 5. Academic Skills Centres (ASC) como ámbito educativo. 6. Una propuesta de actividad didáctica. 7. Conclusiones. 8. Referencias bibliográficas.

Cómo citar: Marzal García-Quismondo, M.A.,Cruz-Palacios, E (2018) Gaming como Instrumento Educativo para una Educación en Competencias Digitales desde los Academic Skills Centres, en Revista General de Información y Documentación 28 (2), 489-506.

\section{Introducción}

Uno de los debates más interesantes que se están planteando en Educación Superior es el modelo de universidad en la Educación del siglo XXI para la Sociedad del conocimiento, en el que la universidad no forme en conocimientos específicos y especializados en una "profesión", lo que impide una adecuada y eficaz planificación del diseño instructivo en los currículos universitarios, sino que faculte en habilidades y competencias en un área de conocimiento determinado, con el fin de preparar al educando en el aprendizaje permanente y colaborativo, correspondiendo a los estudios de posgrado y programas de formación universidadempresa el desarrollo de conocimientos aplicados a un entorno laboral y profesional determinado.

La tendencia plantea la necesidad de inocular en Educación Superior las competencias, especialmente las competencias transversales, base del modelo educativo competencial, que ha tenido como un hito para su diseño e implementación el Proyecto Tuning, con vocación de servir de modelo al Espacio Europeo de Educación Superior (EEES). El modelo educativo se define en el diseño curricular y éste debe plasmarse en los programas didácticos y planes de estudio: en el siglo XXI, conocimiento y cultura son líquidos, el saber es un proceso de aprendizaje continuo, la enseñanza es la creación de escenarios y situaciones para el aprendizaje, el currículo se organiza a partir de competencias transversales.

La propuesta de este modelo educativo competencial en Educación Superior lleva implícita nuevos parámetros didácticos: las aptitudes para la consecución de destrezas (como uso eficaz de las herramientas y técnicas de cada área de conocimiento); capacidades para el logro de habilidades (uso inteligente, con capacidad de diseño y manipulación programada de las herramientas y técnicas); las competencias, en torno a las cuales ha tenido lugar un imprescindible debate (son el núcleo esencial del modelo). Desde nuestra perspectiva, las competencias en Educación son un instrumento para movilizar conocimientos, procedimientos y 
actitudes, desde los contenidos de aprendizaje, para resolver una hipótesis científica o un desafío profesional, otorgando al educando iniciativa, transferencia en el conocimiento e innovación.

Area (2015), relaciona cinco dimensiones de las competencias en Educación, una vez adscritas a la multialfabetización: instrumental (saber acceder y buscar información en diferentes tipos de medios y tecnologías), cognitiva (saber transformar la información en conocimiento), comunicativa (saber expresarse y comunicarse a través de múltiples lenguajes y medios hipermedia y multimedia), axiológica (saber usar la información de manera ética y democrática), emocional (habilidades en el aprendizaje colaborativo). Desde esta óptica, las competencias en Educación se especializan en competencias en información y en comunicación, emergiendo las competencias digitales, fundamento para la Educación Competencial del siglo XXI.

Este contexto define el marco de este estudio, aportando un análisis desde los parámetros de la Documentación como Ciencia: la Enseñanza, mediante plataformas digitales permite incorporar competencialmente los servicios de la web social, los sistemas de gestión del aprendizaje, los portales web educativos e intranets asociadas a Redes Educativas; las competencias digitales como pauta vehicular en el aprendizaje, ampliando el alcance de la alfabetización; los medios didácticos se transforman en materiales didácticos web como libros digitales, Objetos Digitales de Aprendizaje y Recursos Educativos en Abierto, una tendencia para la que este estudio propone el Gaming como objeto educativo y los Academic Skills Centres como el ámbito aplicativo. Sintetizamos la argumentación de este trabajo en la ilustración 1.

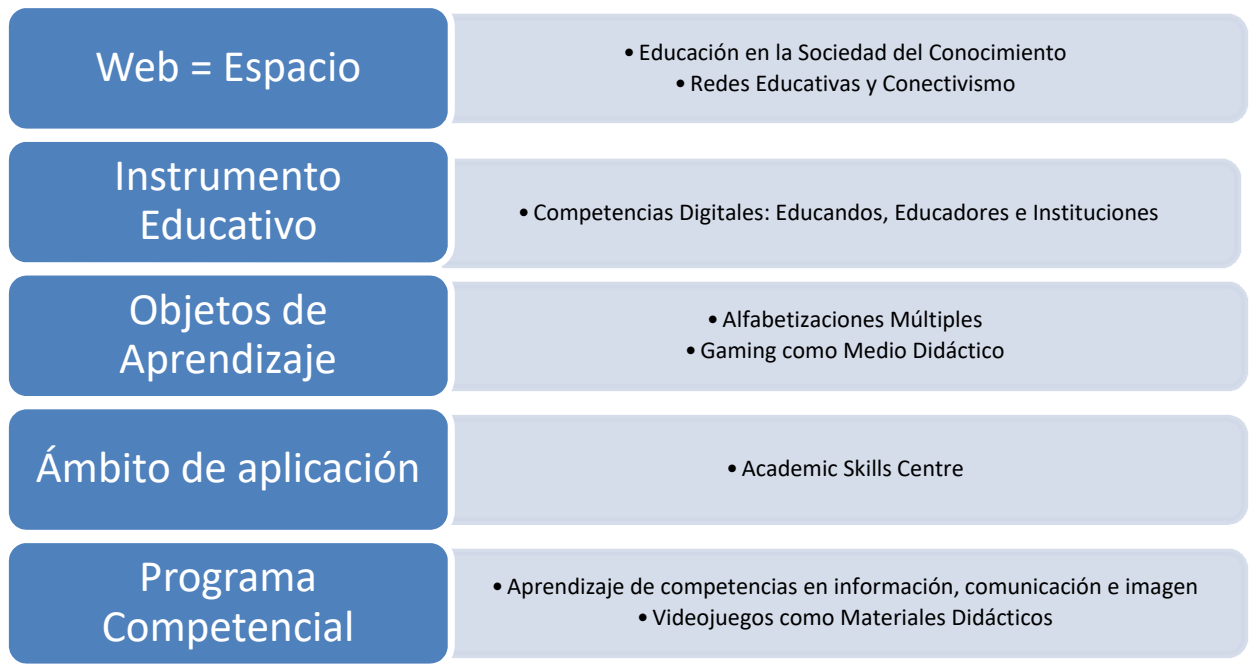

Ilustración 1. Argumentación del artículo en la que se propone un programa competencial que contemple el Gaming para el aprendizaje de competencias digitales a través de los

Academic Skills Centre en la Web. Fuente: elaboración propia 


\section{La web como espacio educativo}

La Educación se proyecta en espacios inmersos en una transformación tecnológica, siendo ejemplo prototípico el Aula 2.0. En este entorno educativo, la innovación educativa aparece como el medio metodológico idóneo para cambiar las prácticas pedagógicas de la cultura líquida y multimodal del siglo XXI, siguiendo la tipificación planteada por Escudero Muñoz (2014): la innovación técnica, referida a las aplicaciones prácticas; la innovación reflexiva, sobre el desarrollo interactivo de los medios y fines didácticos, entrelazando procesos y contenidos; innovación crítica, que incorpora la significación e interpretación de procesos conforme a unos propósitos y valores.

El impacto tecnológico provoca un "comportamiento informacional" en la obtención de conocimiento entre los educandos: prestan más atención a las pantallas que a los libros (Bringué Sala y Sádaba Chalezquer, 2009), poseen más de un dispositivo propio conectado a Internet (Tablet, Smartphone, PC), disponen de multitud de cuentas y perfiles en una diversidad de servicios web (correo electrónico, redes sociales, perfiles web, DNI electrónico, etc.), utilizan dichos dispositivos para todo tipo de consultas e intercambios de información, juegan con diferentes tipos de plataformas de videojuegos y están expuestos a una ingente cantidad de mensajes mediáticos a todas horas en cualquier lugar.

La Educación es un pilar básico de progreso hacia la Sociedad del Conocimiento, que concibe el conocimiento como recurso. Bajo este prisma surge el análisis del informe de Fundación Telefónica (2013), que apunta cinco claves para alcanzar el éxito educativo: a) desarrollar metodologías didácticas bajo el nuevo paradigma digital, primando los objetivos pedagógicos sobre la selección y uso de herramientas web, como también fomentando la inteligencia social a partir de la Web 2.0 y la innovación; b) educar para el bienestar emocional y social, mediante valores, ética y la creatividad, que permita el progreso por estímulos, pensamiento crítico frente a la replicación de lo sabido; c) reconocer el liderazgo de los actores implicados en la educación, como comunidad educativa; d) educar en competencias para el aprendizaje permanente, para que el educando genere sus propias redes de aprendizaje regidas por los principios de interactividad (aprendizaje colaborativo), usabilidad (aprendizaje significativo por uso inteligente de TIC) y relevancia (competencia en la selección de contenidos para un propósito educativo); e) capacitar en el reconocimiento de tendencias.

La Educación del siglo XXI necesita un nuevo modelo pedagógico. El Conectivismo fue presentado por Siemens (2005) como la teoría del aprendizaje que mejor puede responder a los requerimientos de la Sociedad del Conocimiento, en la que el procesamiento e intercambio de información dentro de las redes sociales han hecho replantearse el concepto del aprendizaje. Para Siemens en el proceso del aprendizaje debe primar el valor de lo que se aprende, el fin no debería ser conocer, sino para qué conocer, y esto requiere no solo la adquisición de conocimiento (tarea en la que un ordenador es más eficaz que un ser humano), sino su gestión, que ha de ser eficiente, contextualizada e implicar la evaluación para un conocimiento aplicado. El Conectivismo presenta como principios: a) el 
aprendizaje y el conocimiento se fundamentan en la diversidad de opiniones; b) el aprendizaje es el proceso de conectar nodos y fuentes de información, siendo las conexiones la base del aprendizaje permanente; c) el aprendizaje puede darse en sistemas no humanos; d) la capacidad de conocer es más relevante que lo conocido; e) la comprensión de la interdisciplinariedad y transversalidad de ideas y conceptos es una habilidad básica; f) el aprendizaje conectivista tiene por objetivo el conocimiento preciso y actualizado; g) la toma de decisiones y selección del objeto y objetivos de conocimiento es parte misma del proceso de aprendizaje.

Siemens argumenta que la inteligencia debe referirse al potencial que a cada uno le permiten sus redes, entendidas como la estructura que representa las conexiones con otros, sino también el conjunto de agentes involucrados en la resolución de un problema, la elaboración de un producto tecnológico o la investigación. El flujo de información, muy presente en redes sociales, pasa a ser una clave en la economía del conocimiento. Desde el punto de vista de la información relacional, las propiedades de las interrelaciones pueden ser: jerárquicas; de conectividad o interconexión; derivaciones a partir de una secuencia de fases; de clasificaciones compartidas; o de semejanza entre atributos o valores, además pueden convertirse en datos para realizar un análisis de redes sociales (Hanneman y Riddle, 2005).

Comellas (2010) añadía unos requisitos para que una red sea tal: el conjunto más que la suma de sus partes; identidad propia por símbolos visuales como un logotipo; estructura flexible, pero funcional y estable, con recursos humanos y tecnológicos, adscrita a un contexto y a unos límites; personas; un propósito; autoorganización; intercambio con otras redes; apertura, para incrementar la visibilidad de la influencia sobre otras redes y la sociedad. Las redes presentan claros beneficios como compartir riesgos con otras organizaciones; acceder a nuevos mercados y tecnologías; acelerar el desarrollo de propuestas socioeducativas; aprender de las prácticas innovadoras de otros contextos; y promover la interacción social, la confianza y la reciprocidad para intercambiar conocimientos (Muñoz Moreno, Rodríguez-Gómez y Barrera-Corominas, 2013). Una de las manifestaciones más atractivas de estas redes son las Comunidades Virtuales de Aprendizaje, que tienen por objetivo principal el aprendizaje y el desarrollo de competencias de sus miembros a través de la colaboración.

Redes y Comunidades Virtuales de Aprendizaje necesitan sostenerse en los Entornos Personales de Aprendizaje como conjuntos de herramientas, materiales y recursos humanos que una persona conoce y utiliza para aprender a lo largo de su vida (Marín-Juarros, Negre Bennasar y Pérez Garcías, 2014). En un Entorno Personal de Aprendizaje se necesitan recursos, objetivos y competencias para aprender a aprender. Estas competencias serían aquellas relacionadas con las técnicas de búsqueda y recuperación de información, la gestión de fuentes de información, la lectura digital, el pensamiento crítico, la elaboración de herramientas que faciliten la comprensión, la creación de conocimiento en comunidad, entre otras. Por ello, materializar los Entornos Personales de Aprendizaje implica identificar las personas que pueden ayudarnos (de quién) y con las que compartiremos (con quién); programar los momentos y plazos 
apropiados (cuándo); localizar física y virtualmente el lugar adecuados (dónde); y controlar las herramientas ideales (cómo) mientras nos actualizamos permanentemente. Así se plantean las competencias digitales en Educación.

\section{Competencia digital como instrumento educativo}

Las competencias digitales para Educación se plantean para permitir a los ciudadanos participar en la empleabilidad, la economía, la política, la educación, el ocio y la cultura del siglo XXI. La Unión Europea las consideró como un hito educativo fundamental, y como resultado del Proyecto "DIGCOMP" se ha desarrollado un marco de competencias digitales para los ciudadanos recogido en el informe "DigComp 2.0: The Digital Competence Framework for Citizens. Update Phase 1: The Conceptual Reference Model" (Vuorikari, Punie, Carretero Gomez y Van den Brande, 2016).

Las competencias digitales eran categorizadas en torno a cinco áreas:

Alfabetización en información y datos, cuyas competencias asociadas son: a) navegación con definición de necesidades informativas, búsqueda y filtrado de datos, información y contenidos digitales determinando estrategias propias; b) evaluación de datos, información y contenidos digitales para determinar su fiabilidad conforme a criterios fundados; c) gestión de datos, información y contenidos digitales organizados de modo estructurado.

Comunicación y colaboración, definidas por: a) interacción a través de tecnologías digitales, conforme a un contexto determinado; b) compartir a través de tecnologías digitales datos, información y contenidos digitales; c) desarrollo de la ciudadanía a través de tecnologías digitales; d) colaboración a través de tecnologías digitales para el conocimiento y trabajo compartidos; e) netiqueta, como comportamiento en web; f) gestión de la identidad digital para la reputación e imagen en web.

Creación de contenido digital, mediante: a) desarrollar contenido digital, por edición en distintos formatos; b) integración y reelaboración de contenido digital, por reutilización eficiente; c) uso debido de Copyright y licencias; d) programación de instrucciones comprensibles por un sistema informático para solucionar un problema.

Seguridad, reflejada competencialmente en: a) protección de dispositivos por conocimiento de riesgos y amenazas en los entornos digitales; b) protección de datos personales y de privacidad; c) protección de la salud y del bienestar por inclusión social mediante la inclusión digital; d) protección del medio ambiente.

Resolución de problemas por competencia en la resolución de problemas técnicos, identificación de necesidades y requerimientos tecnológicos (incluida la accesibilidad), uso creativo de las TIC, reconocimiento de la propia "incompetencia" digital.

Este marco es adecuado por fundamentarse en la convergencia de otros quince modelos de competencias digitales, según anota el informe de Ferrari (2012). Si bien estos modelos parecen orientarse hacia los educandos, emergen otros que apelan a 
los educadores, sea el caso del "Marco Común de Competencia Digital Docente" (Instituto Nacional de Tecnologías Educativas y de Formación del Profesorado, 2016), que propone un referente en procesos de formación, evaluación, certificación y acreditación. Registra seis niveles para las competencias digitales (enunciadas como información y alfabetización informacional, comunicación y colaboración, creación de contenidos digitales, seguridad, y resolución de problemas), cada nivel subdividido en dos subniveles etiquetados por unos descriptores que engloban unas capacidades, susceptibles de medir el nivel competencial del docente: A1 y A2 como niveles básicos (para tareas mecánicas y sencillas en actividades concretas), B1 y B2 intermedios (deben aplicarse en contextos para lo cual es necesario un previo análisis utilizando criterios), $\mathrm{C} 1$ y C2 avanzados (planificar, elaborar y aplicar metodologías y estrategias, y evaluarse a sí mismo).

Una inoculación de las competencias digitales en entornos educativos la ofrece el marco propuesto en el informe de la Unión Europea "Promoting Effective Digital-Age Learning: A European Framework for Digital-Competent Educational Organisations" (Kampylis, Punie y Devine, 2015), producto de la convergencia de catorce modelos educativos en contextos europeos y uno estadounidense. Este marco, dirigido a las instituciones educativas, se estructura en ocho elementos temáticos que se dividen en subelementos, con sus descriptores y explicaciones: Liderazgo y Gobernanza; Prácticas de Enseñanza y Aprendizaje; Desarrollo Profesional; Evaluación; Contenido y Currículo; Colaboración y Redes; Infraestructura; Elementos específicos del sector. La progresiva relevancia de las competencias digitales en Educación se debe al impacto en el emprendimiento y la empleabilidad, según recogen Alonso Benito, Fernández Rodríguez, Nyssen González (2009), el "Manifiesto de las Competencias Digitales" de European Schoolnet y Digital Europe en 2016, o el estudio de la Fundación Tecnologías de la Información (2012), con una propuesta de una taxonomía para el ámbito de los contenidos digitales. Al objeto de este trabajo este documento tiene una importancia añadida: el Core pivota sobre lo audiovisual (Animación y VFX; Televisión, Radio y Cine), los videojuegos, la música, las apps, las publicaciones (libros, revistas y periódicos), la publicidad y el social content.

La Educación debe atender al Gaming como medio Didáctico para las Alfabetizaciones Múltiples; considerando el modo de conocer la industria, el videojuego como artefacto cultural y como medio de alfabetizar (consecuencias cognitivas, sensoriales y emocionales de su utilización).

\section{Gaming como objeto educativo}

Una aproximación a este concepto la han ofrecido Kalantzis y Cope (2012), que apuntan los caracteres de una tercera globalización: los significados escritos, visuales y orales comienzan a funcionar juntos en las TIC, de modo que las comunicaciones digitales y las tecnologías de Internet aceleran el proceso de las comunicaciones multimodales. Emerge una nueva conciencia de "actor protagonista" que adquiere la población que ha disfrutado de los videojuegos: el 
trabajo mecánico es para las máquinas, mientras las personas han de asumir labores creativas y de resolución de problemas. Para este desafío aparecen nuevos modelos de alfabetización, cada uno atendiendo a distintos aspectos (mensaje, medio, criterios, procesos cognitivos, metodología y metas pedagógicas, valores y actitudes): la audiovisual, la visual, la digital, la informacional, la transliteracy, la cyberliteracy, la mediática, la Media and Information Literacy (MIL), la New Media Literacy, la metaliteracy, la ludoliteracy, entre otras. La noción de literacy puede ampliarse a una concepción más abstracta y epistemológica, referida a la creación de significados, la evaluación crítica de contextos, la representación del pensamiento, los sistemas de comunicación, la expresión, registro y aplicación del conocimiento y el diseño de los procesos de aprendizaje.

Partiendo de la perspectiva desde la cual entendemos el Gaming (Cruz-Palacios y Marzal García-Quismondo, 2017), es decir, como medio didáctico, debe evaluarse primero la idoneidad de su contenido mediante el sistema de "Pan European Game Information" (PEGI), que consta de una serie de marcas asociadas a los videojuegos que informan de los caracteres de su contenido. Sus beneficios sobre el aprendizaje se evidencian en el estímulo de capacidades de interacción, de motivación, de instrucción de habilidades cognitivas y espaciales, retroalimentación y resolución de problemas, fundamentado por la psicología del aprendizaje social (Etxeberria Balerdi, 2001). Pindado (2005) profundizó los beneficios dependiendo del género: los de acción y arcade desarrollan aspectos sensomotrices (motricidad, agudeza visual, reconocimiento espacial, reflejos y aprendizaje asociativo de procedimientos prácticos), y los de estrategia y simulación más los intelectuales (memoria, atención, pensamiento crítico, razonamiento deductivo y lógico, descubrimiento inductivo, resolución de problemas, toma de decisiones, y creatividad). Un estudio de campo de Connolly, Boyle, MacArthuer, Hainey y Boyle (2012), sobre videojuegos, demuestra que sus beneficios se deben a que la experiencia de uso de los videojuegos es activa, experimental y situacional. Gros (2014) enumera seis tipos de aplicaciones educativas en el ámbito universitario: uso como contexto; desarrollo de competencias; fomento de la motivación y el compromiso; asimilación de contenidos; evaluación de aprendizajes; capacidad en el diseño de juegos.

Respecto a investigaciones más relacionadas con la Alfabetización en Información y las Alfabetizaciones Múltiples, comienza a establecerse un corpus teórico que permite diseñar tanto videojuegos para el aprendizaje de competencias en información como programas competenciales que los integren en el currículo como parte de los materiales didácticos.

Doshi (2006) aporta cuatro ideas para videojuegos en la enseñanza de competencias informacionales. Primero, diseñar juegos "Knowledge Quest" en los que los jugadores deben descubrir conocimiento haciendo uso de recursos bibliotecarios, y juegos de resolución de problemas mediante la consulta de bases de datos especializadas. Segundo, combinar videojuegos con herramientas educativas de enseñanza. Tercero, colaborar con departamentos de informática para el desarrollo de videojuegos. Por último, ayudarse de herramientas de código abierto y las comunidades de desarrolladores para crear videojuegos. 
VanLeer (2006) compara la "narrativa" de tutoriales elaborados por bibliotecas con el diseño de videojuegos, concluyendo que son modelos completamente antagónicos, puesto que los primeros no consiguen el engagement del estudiante para que éste se sienta impulsado o motivado para buscar información. Analiza tutoriales elaborados por bibliotecas, desde la óptica del diseño de videojuegos, destacando aspectos como el diseño de la información, la interactividad, la colaboración con otros usuarios, los "gráficos" y la presencia de elementos lúdicos. Asimismo, provee un listado de recursos útiles para aprender a pensar como un diseñador de juegos cuando se conceptualicen herramientas para la enseñanza de competencias informacionales.

Clyde y Thomas (2008) explicaron conceptos relacionados con los videojuegos (género, narrativa, juego, habilidades, advocacy y persuasión, representación física, y aprendizaje basado en juegos), facilitando su diseño para la mejora de la Alfabetización en Información.

Schiller (2008) enumera una serie de preguntas útiles para diseñar videojuegos cuyos objetivos den lugar a determinados aprendizajes de competencias informacionales:

- ¿Qué quiere el juego que el jugador sea capaz de hacer? (resultado de aprendizaje).

- ¿Qué necesita saber el jugador para hacerlo bien? (currículum).

- ¿Qué actividad facilita el aprendizaje? (pedagogía).

- ¿Cómo demostrará el jugador el aprendizaje? (evaluación).

- ¿Cómo sabrá el juego si el jugador lo ha hecho bien? (criterio).

Gumulak y Webber (2011) entrevistaron a estudiantes para conocer sus aspiraciones motivacionales por los videojuegos y mapearlas con las competencias en información del marco The SCONUL Seven Pillars of Information Literacy.

Jordan (2011), considerando la multimodalidad de recursos de información a la que están expuestos los estudiantes, propone un marco para el "análisis textual" de videojuegos, incluyendo elementos de la teoría de la narrativa en estudios literarios, la retórica y la alfabetización, y considerando la multimodalidad y complejidad presente en el Gaming. Usa este marco para analizar en profundidad el videojuego World of Goo y diseña un curso de Alfabetizaciones Múltiples que permite analizar videojuegos y otros tipos de "textos" (escrito y el audiovisual) como sistemas de información.

Más recientemente, Hale (2018) ha recopilado videojuegos útiles para diferentes áreas de la Alfabetización en Información: Goblin Threat - Plagiarism Game, Gaming Against Plagiarism, I'll Get it!, Within Range, Letterheinz, Library Craft, Quarantined: Axl Wise and the Information Outbreak, Library Scene: Fairfield Edition, The Information Literacy Game, Secret Agents inthe Library, It's alive, Lost in Antarctica, Bioactive, Citation Tic-Tac-Toe, Action Zone's User's Guide to Keyword Challenges / Internet Search Challenge, y Doing Research: An Introduction to the Concepts of Online Research. 
La percepción de la comunidad educativa es muy positiva, tanto por parte de los educandos (Gómez-García, Planells de la Maza y Chicharro-Merayo, 2017), como por parte de los futuros docentes por considerarles un buen medio didáctico (Correa García, Duarte Hueros y Guzmán Franco, 2017). Las instituciones educativas han comenzado a incorporar los videojuegos como una oportunidad: la mediateca del "Meuse Grand Sud", el laboratorio de videojuegos de la "University of California Santa Cruz", la "Kitchener Public Library", el archivo de videojuegos y ordenadores de la biblioteca de la "University of Michigan", el centro de juegos de la biblioteca de la "New York University", la biblioteca de la "Queensland University of Technology", y el museo de videojuegos y consolas "Nostalgia Box".

La aplicación educativa de los videojuegos ha suscitado, en sus efectos, un análisis sobre el comportamiento informacional de los educandos, a través de la Gamificación (Hamari, Koivisto y Sarsa, 2014. Araujo, 2016), como, por ejemplo, el modelo de tipos de jugadores de Marczewski (2015), que establece los siguientes tipos por motivaciones: Jugador (player), la recompensa; Cumplidor (achiever), la maestría; Espíritu libre (free spirit), la autonomía y la autoexpresión; Disruptor (disruptor), el cambio; Filántropo (philanthropist), el propósito y el significado; Socializador (socialiser), la afinidad.

Aceptados por la comunidad educativa y las instituciones educativas, el siguiente paso es determinar cuál es el espacio idóneo donde tratarlos como tal medio y recurso didáctico y donde aplicarlos.

\section{Academic Skills Centres (ASC) como ámbito educativo}

Los videojuegos en Educación, en el entorno de un ambiente Gaming, necesitan un ámbito que los procese como material didáctico web, como Objeto Digital Educativo $\mathrm{u}$ Objeto de Aprendizaje, mediante un procesamiento técnico (identificación, control, representación y etiquetación semántica web), como también los disemine con eficacia educativa (sistema de búsqueda y recuperación conforme a categorías de usos educativos), garantizando su preservación y conservación digitales. Este ámbito, sin duda, son unas bibliotecas universitarias transformadas por la implantación del EEES.

El modelo de inspiración en esta transformación lo ofrecieron los Learning Resources Centre (LRC) de Reino Unido, encargados de fomentar las competencias educativas necesarias mediante la planificación de actividades (Williams y Wavell, 2001). Este modelo originó en España el Centro de Recursos para el Aprendizaje y la Investigación (CRAI), siendo la Red de Bibliotecas Universitarias (REBIUN), dentro de la CRUE, la encargada, mediante la edición de sucesivos planes estratégicos en los que la primera línea estratégica se orienta a los CRAI, de definir el modelo y desarrollarlo. El CRAI quedó definido como "el espacio físico y virtual, flexible, donde convergen y se integran infraestructuras tecnológicas, recursos humanos, espacios, equipamientos y servicios orientados al aprendizaje del alumno y a la investigación" (Domínguez Aroca, 2005, p. 6). 
Los CRAI han ido sufriendo un lento proceso de transformación en su modelo, pero, de nuevo, a partir de los estímulos universitarios anglosajones (un proceso que quedó definido como de "superconvergencia" de servicios), abocan a un cambio por dos factores ya apuntados: el objetivo de la excelencia académica de la universidad y su comunidad educativa, plasmada en los rankings internacionales; la eclosión de la alfabetización académica.

Aparecen los ASC en universidades de Estados Unidos, Australia (Australian National University), Reino Unido, Canadá y Nueva Zelanda (University of Canterbury). Su objetivo es ayudar a los estudiantes en la adquisición de habilidades necesarias para desenvolverse con éxito en el currículo universitario, colaborando en la excelencia académica de sus estudiantes, aumentando su prestigio ante las entidades que invierten en proyectos de investigación gestados en universidades.

En Estados Unidos, en la universidad "Dartmouth Collegue" (Dartmouth Collegue Academic Skills Center), el servicio trata de ayudar a los estudiantes a convertirse en aprendices más eficaces, a través de una amplia variedad de servicios y actividades que son accesibles para todos aquellos que deseen mejorar sus habilidades académicas y de aprendizaje. El ASC enumera un conjunto de razones por las cuales un estudiante podría necesitar sus servicios: mejorar el rendimiento en los exámenes, incomodidad al hablar en clase, falta de comprensión de los puntos fundamentales de las clases presenciales a pesar de asistir a ellas de manera regular, lentitud al leer, dificultad para completar las tareas en el tiempo requerido, invertir mucho tiempo estudiando lenguas extranjeras sin obtener resultados, sentimiento de falta de tiempo, inseguridad en cómo tomar apuntes, querer registrarse para tener un tutor o formar parte de un grupo de estudio, distraerse fácilmente en clase o mientras se estudia.

En Reino Unido, la Universisy of Bath ofrece el servicio ASC a los estudiantes universitarios, a los ya graduados y a los futuros estudiantes. Los programas son desarrollados por un equipo cualificado y experimentado de profesores. Los servicios tratan sobre el pensamiento crítico, evitar el plagio, comunicación escrita y hablada, escritura académica, dar presentaciones y apoyo a la escritura. Ofrecen tutoriales a través de la web y organizan actividades.

En Canadá, la "Trent University" ofrece el servicio, tanto de manera online como en persona, en las áreas de gramática y composición escrita, lectura y pensamiento crítico, investigación y citación, gestión del tiempo, escritura de ensayos científicos e informes de laboratorio, habilidad de escuchar y tomar notas, presentaciones orales, preparación de exámenes, y matemáticas.

Una experiencia muy interesante de estos centros, reconocidos en América Latina como CRA (Centros de Recursos de Aprendizaje), lo ofrece la Universidad Icesi, que lo considera como un centro que apoya a los departamentos académicos en la revisión, ajuste e innovación de las pedagogías y didácticas utilizadas por los profesores, para hacer realidad los lineamientos definidos en el proyecto educativo de la universidad. Además, busca aportar a la comunidad educativa de la región y del país, documentos e información relacionada con la gestión, la innovación y las tendencias en los procesos de enseñanza-aprendizaje, y trata de promover la investigación en el área del aprendizaje y la formación de los docentes en 
estrategias pedagógicas centradas en el aprendizaje activo. Desarrolla los siguientes programas: a) Centro de Escritura, para el desarrollo de las capacidades de análisis, síntesis, solución de problemas y pensamiento crítico de los estudiantes; b) Diplomados, dirigido a los profesores de la universidad que quieran actualizarse en didáctica, evaluación y diseño curricular, para mejorar sus competencias docentes; c) Seminarios; d) Talleres relacionados con la comunicación oral, el uso de TIC en la docencia, el uso de plataformas de e-learning y estrategias didácticas; e) Acompañamiento y asesoría para el aseguramiento del aprendizaje y procesos de autoevaluación. Estos CRA no sustituyen a la biblioteca universitaria, y no se integran como servicio en ella, sino que son centros paralelos que funcionan en el mismo centro educativo.

Una vez demostrado el eficaz impacto de los videojuegos, dentro de una experiencia Gaming, para una educación competencial digital y para el conocimiento en web, así como la emergencia de un espacio idóneo en el tratamiento educativo de estos videojuegos y su ámbito natural para que la experiencia educativa sea más eficaz, los ASC, parece conveniente, como colofón de la argumentación, presentar una propuesta de actividad didáctica bajo estos parámetros.

\section{Una propuesta de actividad didáctica}

Los Objetivos de Aprendizaje son el desarrollo de competencias relativas a la multialfabetización y que se han estructurado en bloques concernientes a la Alfabetización en Información, la Comunicación Web y la Visual Literacy. El método pedagógico es el Gaming, según proponemos en este trabajo. Se trata de envolver a los educandos en situaciones de aprendizaje cuya resolución, proceso seguido en ella y reflexión posterior, inculquen en ellos valores, actitudes, conocimientos y métodos de trabajo considerados propios de las competencias inherentes a una multialfabetización. El videojuego se considera un material didáctico, esto es, un recurso para la generación de las experiencias pedagógicas necesarias para la consecución de las competencias.

Respecto de los medios y tecnología, se utiliza principalmente la Web Social. En un sitio web gestionado por el docente se centraliza toda la experiencia publicándose toda la información sobre ella (fines y actividades pedagógicas, diseño instructivo y resolución de dudas). Se usa un blog individual de cada educando en el que se exponen sus actividades y evidencias de aprendizaje. Docente y discentes también usan el servicio Netvibes para gestionar la publicación de recursos web mediante tecnología RRSS. Se usa una wiki para que los educandos trabajen de manera colaborativa. A su vez, los educandos utilizan una herramienta de publicación de contenidos digitales y/o servicios de redes sociales, a su elección, para narrar el avance de las partidas que realizan con videojuegos elegidos a lo largo de la experiencia educativa. La aplicación de mensajería instantánea WhatsApp sirve como medio para resolución de problemas técnicos. 
El Diseño Instructivo, se presenta: Cronograma y Estructura, la experiencia se desarrolla a lo largo de 12 unidades didácticas (contemplan objetivos de aprendizaje, actividades pedagógicas a realizar por los educandos, entornos de enseñanza, materiales didácticos y evaluación), 1 por semana, las cuales se estructuran en 6 módulos: (0) Reglas y Logística, (1) Videojuegos y Cultura, (2) Alfabetización en Información, (3) Comunicación Web, (4) Visual Literacy, y (5) Videojuegos y Aprendizaje, con una duración de 3 meses.

Para la Dinámica docente, cada unidad didáctica exige una dedicación aproximada de 5 horas de trabajo a la semana, siendo 60 horas el total programado. Son sus principales factores: 1) el anonimato de los educandos, por cuanto todas las cuentas de servicios web utilizadas no contendrán información personal; 2) los educandos son libres de compartir sus proyectos relativos a esta experiencia desde sus cuentas personales; 3) el respeto a los demás es una regla de conducta primordial, por ser un aprendizaje colaborativo e interactivo; 4) las actividades didácticas se editan $\mathrm{y}$ se evalúan en el sitio web del docente (https://gamingformultiliteracies.wordpress.com/); 5) las actividades y tareas son accesibles desde el menú principal, en el nodo "actividades pedagógicas"; 6) todos los sitios web concernientes a esta experiencia educativa se enlazan en la página "Red Educativa" de ese sitio web; 7) la resolución de las actividades se publica en el eportfolio de los educandos, como entradas, y en el blog del docente, utilizándose complementariamente un escritorio en el servicio web "Netvibes", una wiki en el servicio "Wikispaces" y otro medio web a elección de los educandos; 8) la evaluación será conforme a lo trabajado y requerido en las unidades didácticas, un "saber hacer" a partir de un "saber", justificando la evaluación en blog para mejorar el rendimiento; 9) los educandos plantearán sus dudas, o solicitud de recursos complementarios, en el espacio web contemplado en cada unidad didáctica; 10) las entradas de los educandos podrán usar cualquier tipo de contenido: texto, hipertexto, fotografía, esquema, infografía, gráfico, vídeo, etc.; 11) se utiliza un grupo en "WhatsApp" para resolver cuestiones técnicas.

El Diseño de la Programación Didáctica consta:

UD1. Reglas y Logística. Contenidos: 1) Modo de uso del blog donde se explica la dinámica de esta experiencia educativa.; 2) Crear una cuenta y un blog en WordPress.com que se utilizará como eportfolio, con prácticas para aprender a utilizarlo; 3) crear una cuenta en Netvibes.com. y una o dos pestañas para añadir a ella/s los feeds de los blogs de los compañeros y de los comentarios publicados.

UD2. Videojuegos y Cultura. Contenidos: 1) Consulta y análisis de documentos sobre la integración del videojuego en la cultura del siglo XXI como manual teórico: Estudio de la Economía Digital: Los contenidos y servicios digitales, Consultora PWC, 2011; Essential Facts about the computer and video game industry, Sales, Demographic and Usage Data. Entertainment Software Association (ESA), 2017; Libro Blanco del Desarrollo Español de Videojuegos. Asociación Española de Empresas Productoras y Desarrolladoras de Videojuegos y Software de Entretenimiento, 2015; El videojugador español: perfil, hábitos e 
inquietudes de nuestros gamers, AEVI, 2011; ¿Cómo se proyecta el videojuego en el futuro?, Consultora GfK, 2011;2) Experiencias y conductas con videoconsolas y videojuegos, visitando unidades de información como la Videogame Lab de la University California Santa Cruz, Broward County Library, National Videogame Museum, biblioteca de la University of Michigan, Museo de videoconsolas The Nostalgia Box, Centro de juegos de la New York University, Catálogo de las Bibliotecas Públicas del Estado (España); 3) Publicar una entrada en el blog sobre la experiencia con videojuegos propia; 4) Elección de un videojuego aplicable a lo largo de la experiencia educativa, sobre el que se hará la última actividad del curso como exposición oral auxiliándose de tecnologías digitales.

UD3. Necesidades y Fuentes de Información. Contenidos: 1) Consultar información ofrecida por el docente sobre la Alfabetización en Información.; 2) Consultar información sobre las Estrategias de Búsqueda y Fuentes de Información; 3) Analizar los objetivos (principales y secundarios) y todas las posibilidades o modalidades de juego del videojuego elegido (conseguir todos los objetivos y funcionalidades del videojuego, mejorar como videojugador, los beneficios educativos); 4) Publicar los resultados en el blog.

UD4. Estrategias y Evaluación de Búsquedas de Información. Contenidos: 1) Diseñar una estrategia de búsqueda pertinente para localizar las necesidades de información dilucidadas en la Unidad Didáctica 2, analizar los resultados obtenidos y conocer los buscadores y metabuscadores más potentes para mejorar la efectividad de la recuperación; 2) Definir plan de búsqueda, estrategia de recuperación y selección de fuentes idóneas para videojuegos; 3) Publicar los resultados en el blog.

UD5. Uso Ético de la Información. Contenidos: 1) Conocer y comprender los principios y usos de la propiedad intelectual, derechos de autor, y formas de citas, junto con conocimiento de licencias (Creative Commons); 2) Elaborar un repertorio de información recuperada, conforme a estilos de referencia normalizados; 3) Publicar los resultados en el blog

UD6. Gestión de la Información. Contenidos: 1) Comprender los Entornos Personales de Aprendizaje y sus Redes; 2) Organizar el escritorio de Netvibes según los recursos relacionados con los videojuegos; 3) Buscar fuentes de información relacionadas con los videojuegos y las categorías utilizadas previamente en la organización del escritorio Netvibes; 4) Publicar los resultados en el blog.

UD7. Identidad Digital. Contenidos: 1) Explicar en blog los servicios web utilizados, por qué (ámbitos sociales) y cómo (lenguaje y su estilo, formatos, etc.); 2) Comprender los elementos de los perfiles; 3) Seleccionar un "sujeto" de videojuego y buscar sus perfiles en web; 3) Publicar en blog la estrategia de búsqueda de perfiles y analizar la coherencia del perfil con el sujeto. 
UD8. Comunicación en Medios Sociales Web. Contenidos: 1) Factores de la comunicación en web; 2) Evaluación de la comunicación atendiendo a idoneidad del medio (aspectos técnicos disponibles) y aprovechamiento del mismo (aspectos técnicos utilizados), periodicidad de las publicaciones, métodos para codificar el mensaje (texto, imagen, vídeo), lenguaje y su estilo.

UD9. Colaboración para la Construcción de Conocimiento en una Wiki. Contenidos: 1) Construir una wiki sobre videojuegos sobre su definición, historia, formas de clasificación, recursos para su diseño y desarrollo, hábitos de uso, beneficios educativos, buenas prácticas; 2) Desarrollo de modos de creación colaborativa de modos de uso de videojuegos educativos en wiki.

UD10. Concepto y Funciones de la Imagen. Contenidos: 1) Visual Literacy y sus Normas; 2) Aplicación de las Normas a las imágenes de un videojuego para su mayor eficacia educativa; 3) Propuestas de mejora para el aprendizaje.

UD11. Conocer y Saber a través de la Imagen. Contenidos: Planificación de la función educativa de la imagen en un videojuego, cuyos hitos serán: qué y cómo comunicar; qué imágenes seleccionar y cómo; cómo y dónde obtener las imágenes; demostración de observación de derechos de autoría y propiedad intelectual.

UD12. Exposición Oral del Aprendizaje con Videojuegos. Contenido: exposición oral argumentada y explicativa del videojuego que han ido trabajando, auxiliada por las tecnologías digitales. La exposición oral deberá abordar: a) presentación del videojuego en sus caracteres de forma y fondo; b) área de conocimiento sobre la que se aplicará; c) informe de avance en las partidas (objetivos didácticos y competenciales); d) descripción del jugador y sus motivaciones; e) potencial educativo.

\section{Conclusiones}

El análisis realizado busca manifestar cómo la Web debe incorporarse a la Educación como un espacio educativo más: niños y jóvenes obtienen de ella un enorme conjunto de informaciones que las instituciones educativas deben procurar integrar para una obtención eficaz de conocimiento, mediante nuevos medios y métodos, apropiados para los nuevos entornos tecnológicos.

Sin duda, junto a la lecto-escritura de la galaxia Gutenberg, se debe ensayar la lectura digital, muy distintas entre sí. La lectura digital tiene sus propios códigos, estructuras semióticas y semánticas, sus reglas y, sobre todo, descansa sobre una competencia propia en evaluar los contenidos en mosaicos y píldoras de conocimiento (escritos, icónicos, auditivos), propios del hipertexto e hipermedio. Es un espacio educativo donde no se plantea la adquisición de conocimiento, como factor finalista, sino la generación de conocimiento, en constante evolución, 
creación y de forma cooperativa. Sólo así se faculta a los educandos para un aprendizaje permanente y en la actualización de sus competencias profesionales, tal como reclama la Sociedad del conocimiento y sus requisitos en la empleabilidad.

La lectura digital tiene su propia metodología didáctica: el Conectivismo se convierte el modelo pedagógico para aprender en un entorno web basado en el dinamismo de los contenidos, su asociatividad, interactividad, multisecuencialidad y virtualidad; las redes son el medio necesario para un aprendizaje cooperativo y permanente, donde se genera constantemente el conocimiento mediante contenidos digitales que deben ser evaluados para su aplicación; las competencias digitales son las reglas sintácticas y gramaticales para leer digitalmente, aprender en web y publicar conocimiento digital.

Si la metodología didáctica varía, también debe hacerlo el método docente. Las competencias digitales, deben ser el objeto de estudio de una disciplina académica específica, las multialfabetizaciones, expresadas en alfabetización en información, visual literacy, new media literacy, metaliteracy, dependiendo del tipo de contenido digital educativo. Estas disciplinas deben estructurarse conforme a un programa educativo específico: medios e instrumentos didácticos apropiados y aplicables en los ASC; un diseño curricular modular y progresivo, como sólo puede ser aquel cuyo objetivo son las competencias; unos materiales didácticos y objetos de aprendizaje propios, entre ellos los videojuegos educativos expresados en Gaming.

Nos hallamos, pues, ante un modelo educativo competencial que debe ser incorporado a la universidad como educación transversal y no formal, no adscrita a un Departamento o Grado, sino ofrecido como parte del incentivo a la excelencia académica a través de un programa educativo de alfabetización académica, destinado a los alumnos de nuevo ingreso para optimizar su rendimiento académico, y para los alumnos de fin de Grado, Máster o Doctorado, para garantizar su cualificación profesional y científica.

\section{Referencias bibliográficas}

Alonso Benito, L. E.; Fernández Rodríguez, C. J. y Nyssen González, J. Ma (2009). El debate sobre las competencias: una investigación cualitativa en torno a la educación superior $y$ el mercado de trabajo en España. ANECA. $<$ https://dialnet.unirioja.es/servlet/libro? codigo $=405703>$ [Consulta: 31 octubre 2018]

Araújo, I. (2016). Gamification: metodologia para envolver e motivar alunos no processo de aprendizagem. Education in the knowledge society (EKS), 17, (1), 87-108.

Area Moreira, M. (2015). La alfabetización digital y la formación de la ciudadanía del siglo XXI. Rev. de Inv. Educ, 7, (3). <http://www.scielo.org.bo/pdf/rieiii/v7n3/ v7n3_a02.pdf $>$ [Consulta: 31 octubre 2018]

Bringué Sala, X. y Sádaba Chalezquer, C. (2009). La generación interactiva en España: niños y adolescentes ante las pantallas. Barcelona y Madrid: Ariel y Fundación Telefónica.

Clyde, J. y Thomas, C. (2008). Information Literacy Through Digital Games. Comunicación presentada en Information Literacy Uncorked (WILU 37), Kelowna (British Columbia, Canadá). DOI: 10.21083/partnership.v3i2.542. 
Comellas, M. J. (2010). El trabajo en red: un modelo de participación para las familias. Educar, 45, 117-129.

Connolly, T.; Boyle, E.; MacArthuer, E.; Hainey, T. y Boyle, J. (2012). A systematic literature review of empirical evidence on computer games and serious games. Computers \& Education, 59, (2), 661-686.

Correa García, R.; Duarte Hueros, A. y Guzmán Franco, M ${ }^{\mathrm{a}}$ D. (2017). Horizontes educativos de los videojuegos. Propuestas y reflexiones de futuros maestros y educadores sociales. Educar, 53, (1), 67-88.

Cruz-Palacios, E. y Marzal García-Quismondo, M. Á. (2017). Gaming como medio didáctico para las alfabetizaciones múltiples: Videojuegos en la educación del siglo $X X I$. Comunicación presentada en $\mathrm{V}$ Congreso Internacional de Videojuegos $\mathrm{y}$ Educación, Puerto de la Cruz (Santa Cruz de Tenerife, España). $<$ http://hdl.handle.net/10016/25971> [Consulta: 31 octubre 2018]:

Domínguez Aroca, M ${ }^{\mathrm{a}}$ I. (2005). La biblioteca universitaria ante el nuevo modelo de aprendizaje: docentes y bibliotecarios, aprendamos juntos porque trabajamos juntos. RED: Revista de Educación a Distancia, monográfico IV.

Doshi, A. (2006). How Gaming Could Improve Information Literacy. Computers in Libraries, .26, (5). <http://www.infotoday.com/cilmag/may06/Doshi.shtml > [Consulta: 31 octubre 2018]

Escudero Muñoz, J. M. (2014). Contexto, contenidos y procesos de innovación educativa: ¿el dónde y el cómo de la tecnología educativa?. Docencia e Investigación, 24, 13-37.

Etxeberria Balerdi, F. (2001). Videojuegos y educación. Education in the knowledge society $(E K S), \quad(2) . \quad<$ http://campus.usal.es/ teoriaeducacion/rev_numero_02/n2_art etxeberria.htm $>$ [Consulta: 31 octubre 2018]

Ferrari, A. (2012). Digital Competence in Practice: An Analysis of Frameworks. Luxembourg: European Union. DOI: 10.2791/82116.

Fundación Telefónica (2013). 20 claves educativas para el 2020. ¿Cómo debería ser la educación del siglo XXI?. <http://www.fundaciontelefonica.com/arte_cultura/ publicaciones-listado/pagina-item-publicaciones/itempubli/257/> [Consulta: 31 octubre 2018]

Gómez-García, S.; Planells de la Maza, A. J. y Chicharro-Merayo, A. (2017). ¿Los alumnos quieren aprender con videojuegos? Lo que opinan sus usuarios del potencial educativo de este medio. Educar, .53, (1), 49-66.

Gros Salvat, B. (2014). Análisis de las prestaciones de los juegos digitales para la docencia universitaria. Revista Interuniversitaria de Formación del Profesorado, 28, (1), 115 128.

Gumulak, S. y Webber, S. (2011). Playing video games: learning and information literacy. Aslib Proceedings, 63, (2/3), 241-255. DOI: 10.1108/00012531111135682.

Hale, J. (2018). Gaming in der Vermittlung von Informationskompetenz an Hochschulbibliotheken: Chancen und Grenzen gaming-basierter Vermittlungsformen. (Trabajo Fin de Grado, Technical University of Cologne). <https://publiscologne.thkoeln.de/frontdoor/deliver/index/docId/977/file/BA_Hale_Jennifer.pdf $>$ [Consulta: 31 octubre 2018]

Hamari, J.; Koivisto, J. y Sarsa, H. (2014). Does gamification work? - A literature review of empirical studies on gamification. En: Annual Hawaii International Conference on System Sciences : 6 al 9 de enero. Hawaii, 2014, 3025-3034.

Hanneman, R. y Riddle, M. (2005). Introduction to Social Network Methods. $<$ http://faculty.ucr.edu/ hanneman/nettext/> [Consulta: 31 octubre 2018] 
Instituto Nacional de Tecnologías Educativas y de Formación del Profesorado (2016). Marco Común de Competencia Digital Docente 2017 - INTEF. $<$ http://blog.educalab.es/intef/2016/12/22/marco-comun-de-competencia-digitaldocente-2017-intef/> [Consulta: 31 octubre 2018]

Jordan, E. (2011). Place for video games: a theoretical and pedagogical framework for multiliteracies learning in English studies (Tesis Doctoral, Michigan Technological University). $<$ https://digitalcommons.mtu.edu/etds/88/> [Consulta: 31 octubre 2018]

Kalantzis, M. y Cope, B. (2012). Literacies. Cambridge University Press.

Kampylis, P.; Punie, Y. y Devine, J. (2015). Promoting Effective Digital-Age Learning: A European Framework for Digital-Competent Educational Organisations. European Union. DOI: $10.2791 / 54070$.

Marczewski, A. (2015). A Player Framework for Gamification Design. $<$ https://www.gamified.uk/user-types/> [Consulta: 31 octubre 2018]

Marín-Juarros, F.; Negre Bennasar, V. y Pérez Garcías, A. (2014). Entornos y redes personales de aprendizaje (PLE-PLN) para el aprendizaje colaborativo. Comunicar, 42, (21), 35-43.

Muñoz Moreno, J. L.; Rodríguez-Gómez, D. y Barrera-Corominas, A. (2013). Herramientas para la mejora de las organizaciones educativas y su relación con el entorno. Perspectiva educacional, 52, (1), 97-123.

Pindado, J. (2005). Las posibilidades educativas de los videojuegos: una revisión de los estudios más significativos. Pixel-Bit: Revista de medios y educación, 26, 55-67.

Schiller, N. (2008). A portal to student learning: what instruction librarians can learn from video game design. Reference Services Review, 36(4), 351-365.

Siemens, G. (2005). Conectivism: A Learning Theory for the Digital Age. International Journal of Instructional Technology \& Distance Learning, 2, (1).

VanLeer, L. (2006). Interactive Gaming Vs. Library Tutorials for Information Literacy: A Resource Guide. Indiana libraries, 25, (4), 52-55.

Vuorikari, R.; Punie, Y.; Carretero Gomez, S. y Van den Brande, G. (2016). DigComp 2.0: The Digital Competence Framework for Citizens. Update Phase 1: The Conceptual Reference Model. Luxembourg: European Union. DOI: 10.2791/11517.

Williams, D. y Wavell, C. (2001). Evaluating the Impact of the School Library Resource Center on Learning. School Libraries Worldwide, 7, (1), 58-71. 